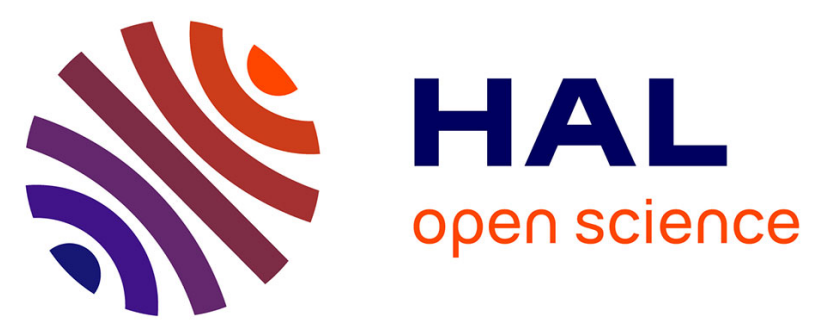

\title{
Resolution power of optical transition radiation: Theoretical considerations
}

Xavier Artru, Robert Chehab, Katja Honkavaara, Alessandro Variola

\section{To cite this version:}

Xavier Artru, Robert Chehab, Katja Honkavaara, Alessandro Variola. Resolution power of optical transition radiation: Theoretical considerations. Nuclear Instruments and Methods in Physics Research Section A: Accelerators, Spectrometers, Detectors and Associated Equipment, 1998, 145, pp.160-168. 10.1016/S0168-583X(98)00402-9 . in2p3-00000007

\section{HAL Id: in2p3-00000007 https://hal.in2p3.fr/in2p3-00000007}

Submitted on 12 Oct 2021

HAL is a multi-disciplinary open access archive for the deposit and dissemination of scientific research documents, whether they are published or not. The documents may come from teaching and research institutions in France or abroad, or from public or private research centers.
L'archive ouverte pluridisciplinaire HAL, est destinée au dépôt et à la diffusion de documents scientifiques de niveau recherche, publiés ou non, émanant des établissements d'enseignement et de recherche français ou étrangers, des laboratoires publics ou privés.

\section{(ㄷ)(1) $\$$}

Distributed under a Creative Commons Attribution - NonCommerciall 4.0 International 


\title{
Resolution power of optical transition radiation: Theoretical considerations
}

\author{
X. Artru ${ }^{\mathrm{a}, *}, \mathrm{R}$. Chehab ${ }^{\mathrm{b}}, \mathrm{K}$. Honkavaara ${ }^{\mathrm{b}, \mathrm{c}}$, A. Variola ${ }^{\mathrm{b}}$ \\ ${ }^{a}$ Institut de Physique Nucléaire de Lyon, IN2P3/CNRS and Université Claude Bernard, Villeurbanne, France \\ b Laboratoire de l'Accélérateur Linéaire, Université Paris Sud, Orsay, France \\ ${ }^{\mathrm{c}}$ Helsinki Institute of Physics, Helsinki, Finland
}

The spatial resolution power of Optical Transition Radiation (OTR), applied to beam profile measurements at high Lorentz factor $\gamma$, is studied taking into account diffraction and self-diffraction effects. Microscopic and macroscopic points of view about the different geometrical natures of the forward and backward OTR sources are presented. Properties of the impact parameter profile $I(\mathbf{b})$ of the OTR emitted by one electron are described. Curves for the modulation transfer function and for the OTR profile of a laminar beam are given. Two methods of improving the resolution are investigated : (a) putting a mask to eliminate small-angle photons, (b) using a polarizer.

\section{Introduction}

The spatial resolution power of Optical Transition Radiation (OTR) for measuring electron beam profiles is still under discussion [1-7]. Invoking self-diffraction related to the narrow angular peak of OTR $(\theta \sim 1 / \gamma)$, a resolution limit $\sim \gamma \lambda$ has been conjectured [1] ( $\lambda$ is the optical wavelength, $\gamma \gg 1$ the Lorentz factor). Even replacing $\gamma \lambda$ by $\gamma \chi$, where $\chi \equiv \lambda /(2 \pi)$ is the reduced wavelength, such a limit would be disastrous at high energy. However this prediction has been

\footnotetext{
${ }^{*}$ Corresponding author. Tel.: 33472 431061; fax: 33472 448004;; e-mail: x.artru@ipnl.in2p3.fr
}

questioned by Rule and Fiorito [2]. These authors calculated the spatial OTR profiles, including diffraction, in the scalar wave theory and showed that the resolution limit is not very different from the intrinsic one of the optical system, $\Delta \mathbf{b} \sim \lambda / \theta_{1}$, where $\theta_{1}$ is the optical aperture. Then the resolution would be acceptable at any energy. Jenkins [3] and Lebedev [4] studied the same problem in the vector theory, thus taking into account the radial polarization of transition radiation. We quote Lebedev's result for the full width at half-maximum (FWHM)

$$
\Delta \mathbf{b}_{\mathrm{FWHM}} \simeq 1.44 \lambda / \theta_{1} \quad\left(\text { for } \theta_{1} \gg \gamma^{-1}\right) .
$$

This value is about 2 times larger than in the scalar theory, but independent of gamma and only 3 
times the usual value for a point source $\left(0.51 \lambda / \theta_{1}\right)$, supporting the opinion that the resolution is essentially governed by the optical system. However, Jenkins and Lebedev also pointed out the existence of a halo of diameter $\sim \gamma \lambda$ which, according to Lebedev, "carries much larger energy than the central spot" and "makes a measurement of the beam halo difficult". From the experimental side, beam RMS widths smaller than $\gamma \chi$ by factors 2 [5], 3 [6] or 5 [7] have been measured with OTR.

In the following, we will try to clarify the question of the resolution power using physical arguments and not too complicated mathematics. We will also indicate what factors can influence or improve the resolution: formation zone effects, diaphragm, masks, polarizers and apodization.

\section{Angular and spatial distribution of OTR}

The distribution of transition radiation photon with momentum $\mathbf{k}$ and polarization $\hat{\mathbf{e}}$ is given [810] by

$\mathrm{d} N_{\hat{\mathbf{e}}}=\left(16 \pi^{3}\right)^{-1} \omega \mathrm{d} \omega \mathrm{d} \Omega\left|a_{\hat{\mathbf{e}}}\right|^{2}$,

with

$$
\begin{aligned}
a_{\perp}= & \pm \mathrm{i} e(\hat{\mathbf{y}} \cdot \mathbf{v})\left[\frac{1}{\omega-\mathbf{v} \cdot \mathbf{k}}-f_{\perp} \frac{1}{\omega-\mathbf{v} \cdot \mathbf{k}^{\prime}}\right. \\
& \left.+r_{\perp} \frac{1}{\omega-\mathbf{v} \cdot \mathbf{k}^{\prime \prime}}\right] \\
a_{\|}= & \pm \mathrm{i} e(\hat{\mathbf{y}} \times \mathbf{v}) \cdot\left[-\frac{\hat{\mathbf{k}}}{\omega-\mathbf{v} \cdot \mathbf{k}}+f_{\|} \frac{\hat{\mathbf{k}}^{\prime}}{\omega-\mathbf{v} \cdot \mathbf{k}^{\prime}}\right. \\
& \left.+r_{\|} \frac{\hat{\mathbf{k}}^{\prime \prime}}{\omega-\mathbf{v} \cdot \mathbf{k}^{\prime \prime}}\right] .
\end{aligned}
$$

The overall + or - signs correspond to forward or backward geometry. The three terms in the square brakets correspond to "direct", "refracted" and "reflected" waves. Considering a gedanken wave of momentum $-\mathbf{k}$ coming from the detector, $-\mathbf{k}^{\prime}$ and $-\mathbf{k}^{\prime \prime}$ are the momenta after refraction and reflection, respectively. Eqs. (3a) and (3b) correspond to the polarizations vectors $\hat{\mathbf{e}}_{\perp}=\hat{\mathbf{y}}$ and $\hat{\mathbf{e}}_{\|}=\hat{\mathbf{y}} \times \hat{\mathbf{k}}$, where $\hat{\mathbf{y}}$ is the unit vector normal to the $\left(\mathbf{k}, \mathbf{k}^{\prime}, \mathbf{k}^{\prime \prime}\right)$ plane. $f_{\|}, r_{\|}, f_{\perp}$ and $r_{\perp}$ are the Fresnel coefficients for the gedanken wave (there are two conventions for the sign of $r_{\|}$. We use the one in which $r_{\|}=+r_{\perp}$ at normal incidence). Vectors with a "hat" are unitary, for instance $\hat{\mathbf{k}} \equiv \mathbf{k} /|\mathbf{k}|$. In our units, $\hbar=c=1, e^{2} /(4 \pi) \equiv \alpha=1 / 137$.

In the case of forward OTR, the direct term dominates and yields [11]

$I(\omega, \theta)=\omega \frac{\mathrm{d} N}{\mathrm{~d} \omega \mathrm{d} \Omega} \simeq \frac{\alpha}{\pi^{2}}\left(\frac{\theta}{\gamma^{-2}+\theta^{2}}\right)^{2}$

$(\gamma \gg 1, \theta \ll 1)$,

$\theta$ being the angle between $\mathbf{k}$ and $\mathbf{v}$. This is the spectrum emitted by a suddenly accelerated electron. The same formula applies to backward OTR [8] on a perfectly reflecting metal $\left(r_{\|}=r_{\perp}=-1\right)$. In this case the reflected term dominates and $\theta$ is the angle between $\mathbf{k}$ and the specular direction $\hat{\mathbf{v}}_{\text {spec }}$ of $\hat{\mathbf{v}}$. Eq. (4) is now the spectrum emitted by a suddenly stopped "image positron". The radiation field (in the far-field region) can be decomposed in plane waves

$\mathbf{E}(t, \mathbf{r})=\int \frac{\mathrm{d}^{3} \mathbf{k}}{(2 \pi)^{3}} \tilde{\mathbf{E}}(\mathbf{k}) \mathrm{e}^{\mathrm{i} k \cdot \mathbf{r}-\mathrm{i}|\mathbf{k}| t}$.

with

$\tilde{\mathbf{E}}(\mathbf{k}) \simeq+\mathrm{i} e \frac{\mathbf{k}_{T}}{q_{0}^{2}+\mathbf{k}_{\mathrm{T}}^{2}}$,

and $q_{0} \equiv k_{L} \gamma^{-1} \simeq \omega \gamma^{-1}$. This field resembles the relativistic Coulomb field of the electron involved in the method of quasi-real photons [12]. The latter is given by Eq. (6) and

$\mathbf{E}(t, \mathbf{r})=\int \frac{\mathrm{d}^{3} \mathbf{k}}{(2 \pi)^{3}} \tilde{\mathbf{E}}(\mathbf{k}) \mathrm{e}^{\mathrm{i} k \cdot \mathbf{r}-\mathrm{i}(\mathbf{k} \cdot \mathbf{v}) t}$

and has frequencies $\omega=\mathbf{k} \cdot \mathbf{v}$ (virtual photons), while the OTR radiation field has frequencies $\omega=|\mathbf{k}|$ (real photons).

Let us first consider backward OTR. From the microscopic point of view, it is generated coherently by the collective motion of the metallic electrons in response to the transient Coulomb field of the incident particle. It is therefore a transversally extended source. Its impact parameter profile is obtained by squaring the Fourier 
transform of $\hat{\mathbf{E}}(\mathbf{k})$ with respect to $\mathbf{k}_{\mathrm{T}}$ at fixed $k_{L} \simeq \omega$

$$
\begin{aligned}
I(\omega, \mathbf{b}) & \equiv \frac{\omega \mathrm{d} N}{\mathrm{~d} \omega \mathrm{d}^{2} \mathbf{b}} \simeq \pi^{-1}|\mathbf{E}(\omega, \mathbf{b})|^{2} \\
& =4 \alpha\left|\int \frac{\mathrm{d}^{2} \mathbf{k}_{\mathrm{T}}}{(2 \pi)^{2}} \mathrm{e}^{\mathrm{i} k_{\mathrm{T}} \cdot \mathbf{b}} \frac{\mathbf{k}_{\mathrm{T}}}{q_{0}^{2}+\mathbf{k}_{\mathrm{T}}^{2}} \Theta\left(q_{1}-\left|\mathbf{k}_{\mathrm{T}}\right|\right)\right|^{2},
\end{aligned}
$$

$\Theta$ being the step function and $q_{1} \equiv \omega \theta_{1}$ the limit imposed by the entrance diaphragm. This profile takes into account self-diffraction as well as the diffraction by the optical apparatus; it is the one which is observed in the image plane for a magnification equal to unity. For $q_{1} / q_{0}=\gamma \theta_{1} \gg 1$, we have:

$I(\mathbf{b}) \simeq \frac{\alpha}{\pi^{2}}\left|q_{0} K_{1}\left(q_{0} b\right)-b^{-1} J_{0}\left(q_{1} b\right)\right|^{2}$,

where $J_{0}$ and $K_{1}$ are Bessel functions of first and second kind. This formula generalizes Eq. (22) of Lebedev's paper to all values of $b$. The genuine OTR profile, with only self-diffraction, could be obtained from Eq. (8) by replacing the $\Theta$-function with some smooth cut-off at $q_{1} \sim \omega$. From now on we write $I(\mathbf{b})$ instead of $I(\omega, \mathbf{b})$. Introducing the two characteristic distances

$b_{0}=1 / q_{0}=\gamma \chi, \quad b_{1}=1 / q_{1}=\chi / \theta_{1}$,

we have phenomenologically

$I(\mathbf{b}) \sim \frac{\alpha}{\pi^{2} b^{2}} \Theta\left(b-b_{1}\right) \Theta\left(b_{0}-b\right)$.

These cutoffs prevent logarithmic divergences of the radiated energy. Due to the rotational invariance of Eq. (8) and the vector nature of the field, $\mathbf{E}(\omega, \mathbf{b})$ vanishes at $\mathbf{b}=0$. It makes the FWHM larger than in the scalar theory. The shape of $I(b)$ in the peak region is shown in Fig. 1.

From the macroscopic point of view, backward OTR can be considered as the metallic reflection of the quasi-real photons, which become real after reflection. In fact, Eqs. (6), (8) and (9) hold for the decomposition of the relativistic Coulomb field into quasi-real photons [12-14], but with $q_{1}=\infty$ and discarding the $J_{0}$ term of Eq. (9). The remaining term $q_{0} K_{1}\left(q_{0} b\right)$ is singular at $\mathbf{b}=0$. This singularity is related to the arbitrary large trans-

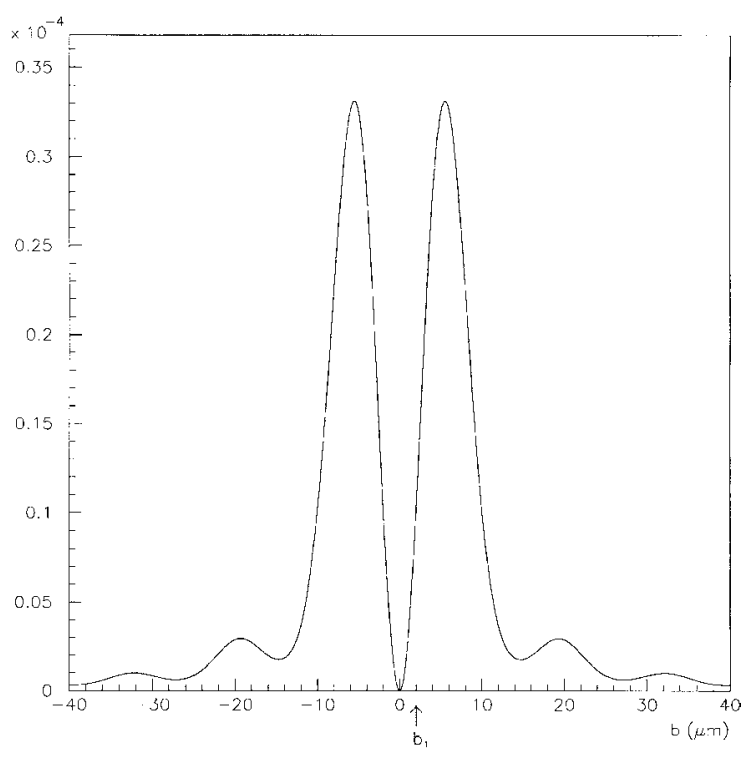

Fig. 1. Ordinary OTR profile according to Eqs. (8) and (9) with $\gamma m_{e}=6.4 \mathrm{GeV}, \lambda=500 \mathrm{~nm}, \theta_{1}=0.04 \mathrm{rad}\left(q_{0}=1.0 \mathrm{~mm}^{-1}\right.$, $\left.q_{1}=0.5 \mu \mathrm{m}^{-1}\right)$. The vertical scale is in $\mu \mathrm{m}^{-2}$.

verse momenta of the virtual photons, whereas the real ones are bounded by $\omega$.

The above microscopic and macroscopic points of view are not easily transposable to forward OTR. I(b) defined by Eq. (8) does not represent the spatial energy flux in the vicinity of the surface. In fact, the formation zone, roughly defined by

$b \lesssim b_{0}, \quad l \lesssim \chi \gamma^{2}$

is rather depleted of electromagnetic flux, due to the absorption or screening of the quasi-real photons inside the material. Besides, forward OTR does not need a reflecting or sharp planar boundary. Thus forward OTR should rather be considered as emitted by a longitudinally extended source (the outgoing electron current). Nevertheless, if one collects the light in the "far-field" region, where Eqs. (5)-(7) are valid, one obtains in the image plane of the radiator the same profile I(b) as for backward OTR, i.e. Eqs. (8) and (9).

\section{Formation zone effects}

In the case of backward OTR, Eqs. (4), (6), (8) and (9) are obtained only if the electron motion is 
in vacuum, rectilinear and uniform over a distance large compared to the formation length,

$$
l_{\mathrm{f}}(\theta) \sim \frac{2 \chi}{\gamma^{-2}+\theta^{2}} .
$$

At high energy, $l_{\mathrm{f}}\left(\gamma^{-1}\right)=\chi \gamma^{2}$ is very large and can even exceed the laboratory size. If the beam is bent or if it traverses an opaque material within this distance before the foil, the peak at $\theta=\gamma^{-1}$ is absent and replaced by a wider structure. Accordingly, the measured intensity profile can be narrower than $b_{0}$. One can treat this effect by considering the radiation coming from the upstream apparatus: synchrotron radiation for a bending magnet or forward OTR for an opaque material. Whatever it is, this radiation is reflected by the foil and interfere with the OTR radiation from the foil itself. This effect is at the basis of the Wartski interferometer [8]. Another point of view, more phenomenological, is that the upstream apparatus removes the quasi-real photon cloud and it takes a length $\sim l_{\mathrm{f}}(\theta)$ to re-create the quasi-real photons of angle $\theta$. The same kind of phenomenon should occur when the upstream beam passes at distance smaller than $b_{0}$ to a piece of matter, producing diffraction radiation and scraping out part of the quasi-real photon cloud.

Similar considerations can be made in the case of forward OTR. To avoid formation zone effects, one must in particular put the collecting mirror at distance larger than $l_{\mathrm{f}}(\theta)$. Otherwise, transition or diffraction radiation will be produced on the collecting mirror and interfere with forward OTR.

Contrary to forward OTR, backward OTR, can be collected very close to the foil if the latter is inclined with respect to the beam. If the foil is perpendicular to the beam, as in Fig. 1 of Ref. [3], the collecting mirror may encroach on the formation zone.

Radiation formulas (2)-(6) work in the far-field region. In both forward and backward OTR, the "near-field region" is given by

$l_{\text {near }}(\psi)=\frac{1}{\omega-\mathbf{v} \cdot \mathbf{k}}=\frac{\chi}{1-v \cos \psi}$,

$\psi$ being the angle between the detector direction and the electron velocity. For the backward case, $\psi \sim 1$ and $l_{\text {near }} \ll l_{\mathrm{f}}$. For the forward case, $\psi \equiv \theta$, therefore $l_{\text {near }} \equiv l_{\mathrm{f}}$ and the near-field region coincides with the formation zone. So far-field conditions do not seem to bring new constraints compared to formation zone conditions.

\section{The spatial resolution of ordinary OTR}

Let us now study the spatial resolution in the idealized case with no formation zone effect. Focusing the optical system on the foil, the observed profile is the profile of the electron beam convoluted with $I(\mathbf{b})$. As we have seen, there are two very different characteristic scales, $b_{0}$ and $b_{1}$ and it is not easy to say a priori which one is relevant.

If one only wants to measure the FWHM of the beam, then the resolution limit is given by the FWHM of $I(\mathbf{b})$, of the order of $10 b_{1}$ (see Eq. (1) and Fig. 1). One may however ask for more detailed informations such as

- the degree of uniformity of the beam density,

- the sharpness of the beam contour and the importance of the halo.

The visibility of beam nonuniformities depends on the modulation transfer function,

$\operatorname{MTF}(q) \equiv \tilde{I}(\mathbf{q}) / \tilde{I}(0)$,

with

$\frac{\tilde{I}(\mathbf{q})=\int \mathrm{d}^{2} \mathbf{b} \mathrm{e}^{-\mathrm{i} \boldsymbol{q}} \cdot \mathbf{b} I(\mathbf{b})=\pi^{-1} \int_{D} \mathrm{~d}^{2} \mathbf{k}_{\mathrm{T}}}{(2 \pi)^{2} \tilde{\mathbf{E}}\left(k_{\mathrm{L}}, \mathbf{k}_{\mathrm{T}}\right) \cdot \tilde{\mathbf{E}}^{*}\left(k_{\mathrm{L}}, \mathbf{k}_{\mathrm{T}}-\mathbf{q}\right),}$

where the integration is restricted to the domain $D$ defined by $\left|\mathbf{k}_{\mathrm{T}}\right| \leqslant q_{1},\left|\mathbf{k}_{\mathrm{T}}-\mathbf{q}\right| \leqslant q_{1}$ and $\tilde{\mathbf{E}}\left(k_{\mathrm{L}}, \mathbf{k}_{\mathrm{T}}\right)$ is given by Eq. (6). We have

$\tilde{I}(0)=\frac{\omega \mathrm{d} N}{\mathrm{~d} \omega} \simeq \frac{\alpha}{\pi}\left[2 \ln \left(\gamma \theta_{1}\right)-1\right]$.

The MTF function is shown in Fig. 2. It vanishes for $q \geqslant 2 q_{1}$ and can be approximated by

$\operatorname{MTF}(q) \simeq$

$\begin{cases}1 & \text { for } q \leqslant \mathrm{e}^{0.5} q_{0}, \\ \ln \left(q_{1} / q\right) / \ln \left[q_{1} /\left(\mathrm{e}^{0.5} q_{0}\right)\right] & \text { for } \mathrm{e}^{0.5} q_{0} \leqslant q \leqslant q_{1}, \\ 0 & \text { for } q \geqslant q_{1} .\end{cases}$ 


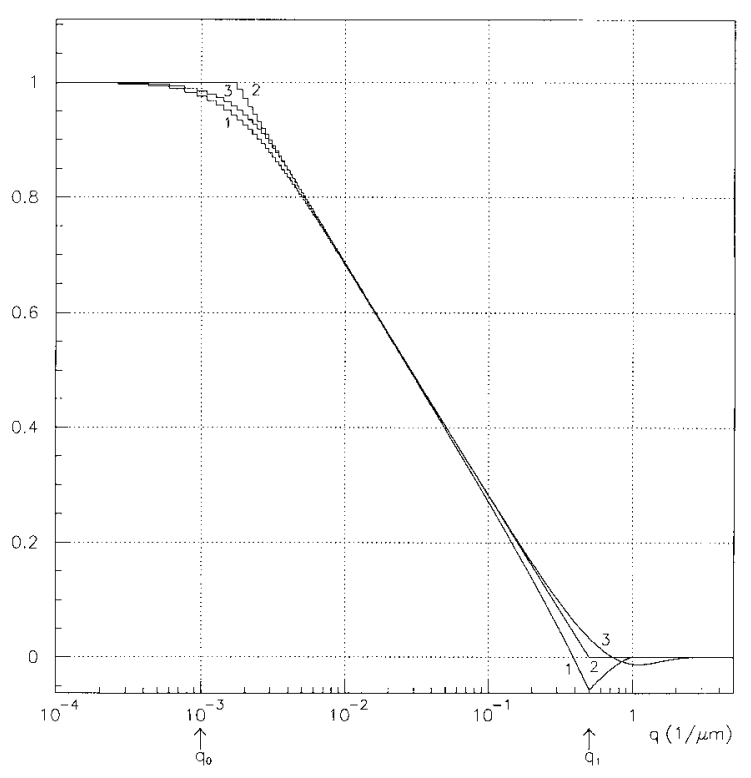

Fig. 2. MTF function for ordinary OTR: (1) Exact form, Eqs. (15) and (16); (2) appromation of Eq. (18); (3) apodized form, from Eq. (28). The parameters are the same as in Fig. 1.

The MTF gives the contrast of the images of beam granularities of size $\delta r \sim q^{-1}$, but assuming a beam radius $R \gg b_{0}$. Let us instead consider the case $R \lesssim b_{0}$, more realistic at high $\gamma$. The illumination of the beam image is on the average

$$
\langle L\rangle \sim\left\langle\rho_{e}\right\rangle \int_{b<R} \mathrm{~d}^{2} \mathbf{b} I(\mathbf{b})
$$

and it fluctuates by

$\delta L \sim\left\langle\rho_{e}\right\rangle \int_{b<\delta r} \mathrm{~d}^{2} \mathbf{b} I(\mathbf{b})$,

$\rho_{e}$ being the beam transverse density. Using approximations (11) and (18), one obtains the contrast:

$$
\frac{\delta L}{\langle L\rangle} \sim \frac{\ln \left(\min \left\{\delta r, b_{0}\right\} / b_{1}\right)}{\ln \left(\min \left\{R, b_{0}\right\} / b_{1}\right)} \sim \frac{\operatorname{MTF}\left(\delta r^{-1}\right)}{\operatorname{MTF}\left(R^{-1}\right)} .
$$

Note that this equation also applies to the case $R>b_{0}$ as well.

In conclusion the visibility of the substructure depends not only on $\delta r$ but also on $R$ and the contrast sensitivity of the camera (including background noise, threshold and saturation ef- fects). For instance, a camera sensitive to a contrast ratio $(\langle L\rangle+\delta L):(\langle L\rangle-\delta L)=5: 3$ can measure substructures of size $\delta r \sim b_{1}^{3 / 4}$ $\times \min \left\{R, b_{0}\right\}^{1 / 4}$, a camera sensitive to a contrast ratio of 11:9 can measure substructures of size $\delta r \sim b_{1}^{0.9} \times \min \left\{R, b_{0}\right\}^{0.1}$, etc. These estimates indicate only the order of magnitude of $\delta r$. Precise values for specific experimental conditions should be better obtained by numerical simulations.

\section{Effect of a central mask}

Looking at Eq. (11), one can crudely simulate the OTR spot by a superposition of concentric halos of equal flux but radiuses increasing in geometric progression, e.g.,

$I(\mathbf{b}) \sim \frac{\alpha}{\pi^{2}} \sum_{n=0}^{N} b_{n}^{-2} \Theta\left(b_{n}-b\right)$,

with $b_{n} \sim b_{1} \mathrm{e}^{n / 2}$ and $N \sim \ln \left(b_{0}^{2} / b_{1}^{2}\right)$. Each halo carries the flux $\omega \mathrm{d} N / \mathrm{d} \omega=\alpha / \pi$. According to the uncertainty principle, the halo of size $b_{\mathrm{n}}$ is made by photons emitted at angle $\theta \sim \chi / b_{n}$. It is possible to suppress the largest halos, thus improving the spatial resolution, by putting a mask inside the optical system which absorbs the photons up to some angle $\theta_{m} .{ }^{1}$ It mimics an increase of $q_{0}$, i.e., a decrease of $\gamma$ and $b_{0}$. The total flux is reduced only by the ratio $\ln \left(\theta_{1} / \theta_{m}\right) / \ln \left(\gamma \theta_{1}\right)$. The "masked" OTR profile is obtained by inserting a second step function $\Theta\left(\left|\mathbf{k}_{\mathrm{T}}\right|-q_{m}\right)$ in Eq. (8). For $\gamma^{-1} \ll \theta_{m} \ll$ $\theta_{1}$, it is given by:

$$
\begin{aligned}
I(\mathbf{b}) & \simeq \frac{\alpha}{\pi^{2} b^{2}}\left|J_{0}\left(q_{m} b\right)-J_{0}\left(q_{1} b\right)\right|^{2} \\
& \sim \frac{\alpha}{\pi^{2} b^{2}} \Theta\left(b-b_{1}\right) \Theta\left(b_{m}-b\right),
\end{aligned}
$$

with

$q_{m}=\omega \theta_{m}=1 / b_{m}, \quad b_{m}=\chi / \theta_{m}$.

Fig. 3 compares the "masked" $b^{2} I(\mathbf{b})$ to the unmasked one for $\theta_{m}=2.0\left(\gamma^{-1} \theta_{1}\right)^{1 / 2}$.

\footnotetext{
${ }^{1}$ This method has already been proposed in Refs. $[3,5,11,15]$.
} 


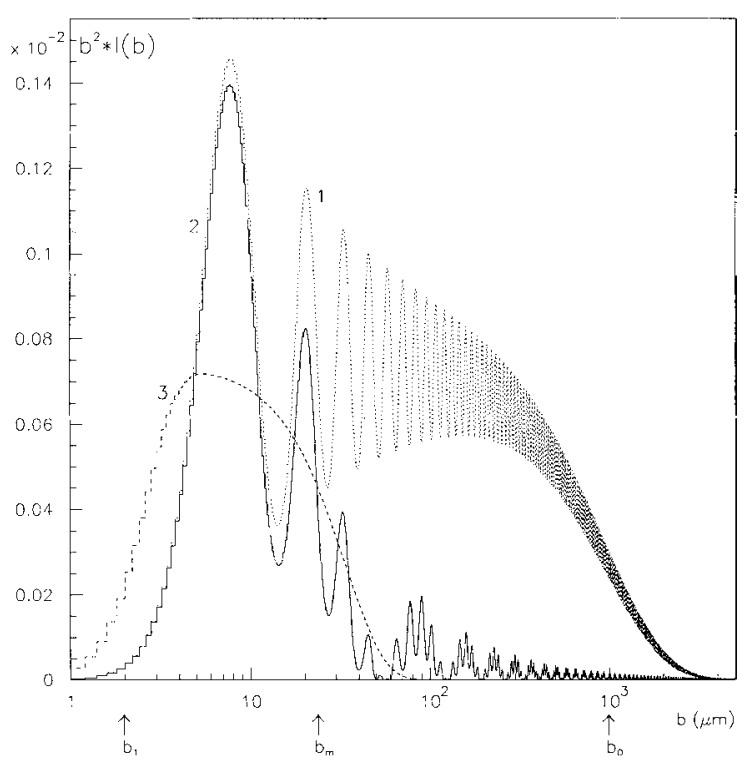

Fig. 3. Effect of a mask on the OTR profile. The curves show $b^{2} I(\mathbf{b})$, with $b$ in logarithmic scale: (1) without mask; (2) with a mask at $\theta_{m}=3.6 \mathrm{mrad}\left[q_{m}=2.0\left(q_{0} q_{1}\right)^{1 / 2}\right] ;$ (3) with apodized mask and diaphragm (see Section 7). Other parameters are the same as in Fig. $1 . b_{0}, b_{1}$ and $b_{\mathrm{m}}$ are indicated by arrows.

The mask should in principle be put in the image focal plane (see Fig. 4(a)), but probably it might also be put on the collecting lens if the radius of the mask is much larger than both $b_{0}$ and the beam radius. A mirror can be placed in front of the mask, deflecting the small-angle photons for beam divergence studies. One may also separate the small-angle and large-angle photons using a mirror with a hole, as in Fig. 4(b) (see also Ref. [3]). In Ref. [7], the small-angle photons were avoided by the collecting mirror placed on one side of beam axis.

\section{Effect of a polarizer}

OTR has a "natural" polarization parallel to $\mathbf{k}_{\mathrm{T}}$ in momentum space and $\mathbf{b}$ in impact parameter space. When one selects, e.g., the $y$-component with a polarizer, then $I(x, y)$ is multiplied by $y^{2} /\left(x^{2}+y^{2}\right)$. The width of the OTR spot is therefore reduced in the $x$ direction. Fig. 5 shows the one-dimensional profiles

$$
\begin{aligned}
& J_{x}(x)=\int \mathrm{d} y I(\mathbf{b}) \frac{x^{2}}{b^{2}}, \\
& J_{y}(x)=\int \mathrm{d} y I(\mathbf{b}) \frac{y^{2}}{b^{2}}
\end{aligned}
$$

and $J(x)=J_{x}(x)+J_{y}(x)$ for the $x$-, the $y$ - and the natural polarizations, respectively. One can infer that two orthogonally polarized OTR images give a significantly better resolution in $x$ and $y$ than one image without polarizer.

Fig. 6 shows the one-dimensional OTR profiles in $x$ of a beam whose density is uniform in the window $[-50 \mu \mathrm{m},+50 \mu \mathrm{m}]$. They are obtained by convoluting the window function with
(a) $J(x)$ for ordinary OTR
(b) $J(x)$ with a mask,
(c) "unmasked" $J_{y}(x)$,
(d) "masked" $J_{y}(x)$.

All these profiles have FWHM widths close or equal to the electron beam one: 105.4, 100, 102.7 and $100 \mu \mathrm{m}$ respectively for a, b, c and d. Thus ordinary OTR is good enough for measuring the FWHM, in spite of the fact that $\gamma \chi$ is 10 times larger. However the "unmasked" profiles (a and c) have important tails which could mimic a beam halo. These tails are strongly reduced for masked OTR. One also sees that the sharpness of the edges receives cumulative improvements from the mask and the polarizer. Defining

$$
\begin{aligned}
\delta r= & (\text { radius at } 1 / 4 \text { maximum }) \\
& -(\text { radius at } 3 / 4 \text { maximum }),
\end{aligned}
$$

we obtain $\delta r \simeq 19,11,14$ and $6.5 \mu \mathrm{m}$ for cases (a), (b), (c), and (d), respectively.

\section{Apodization}

The sharp cutoffs in $\theta$ made by the diaphragm and the mask result in oscillations and a not very fast decrease of $I(\mathbf{b})\left(\sim b^{-3}\right.$ for $\left.b \gg b_{m}\right)$. Also the integrals for $\langle|\mathbf{b}|\rangle$ and $\left\langle\mathbf{b}^{2}\right\rangle$ diverge. These oscillations are suppressed and $I(\mathbf{b})$ decreases faster (apodization) if one replaces the sharp-edged mask and diaphragm by a window of smoothly varying transparency. Accordingly the step functions in Eq. (8) are replaced by a smooth pupil function, for instance 
a)

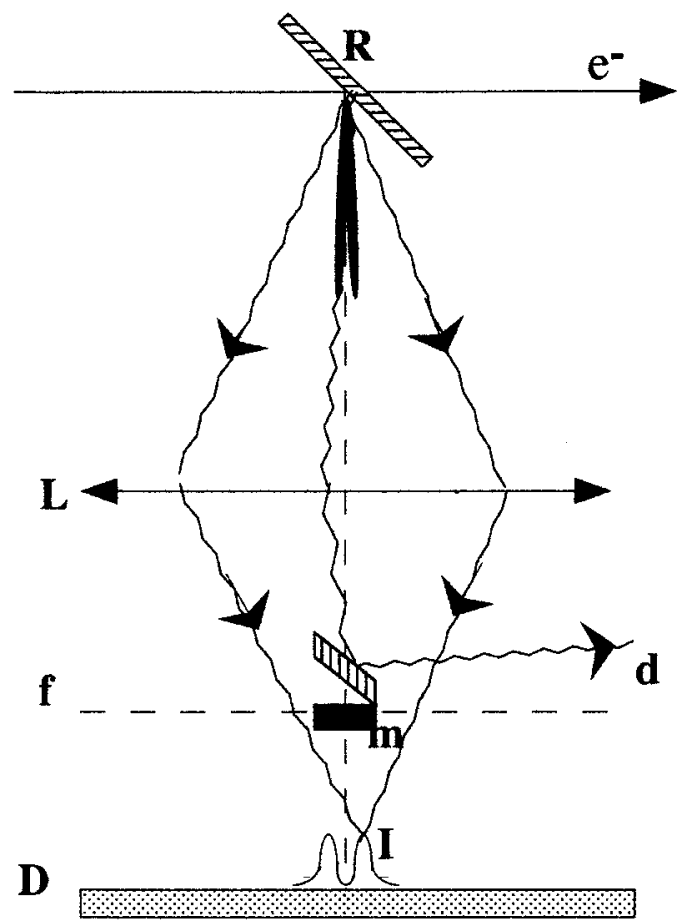

b)

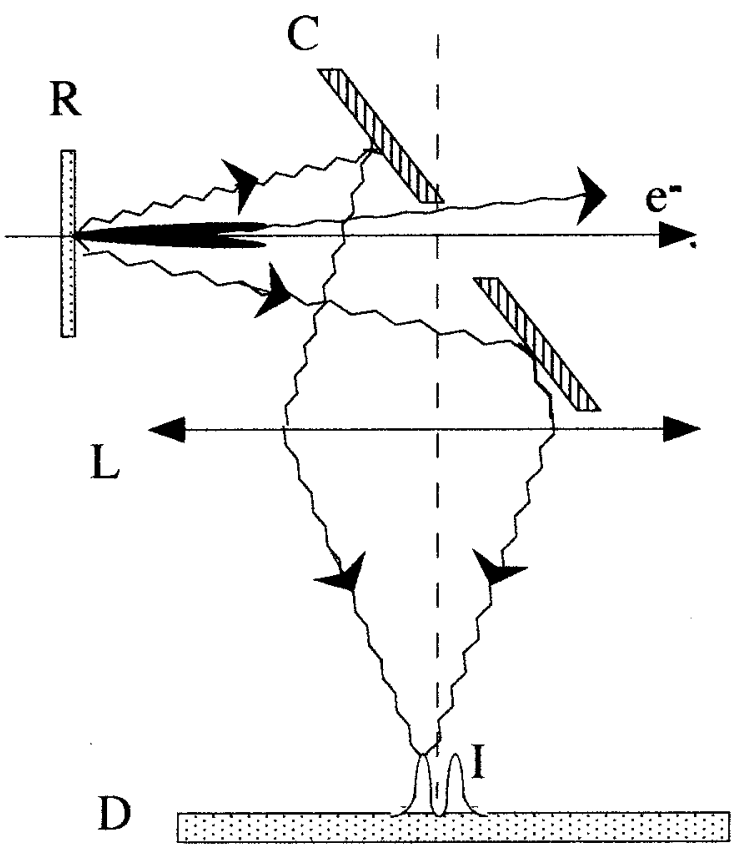

Fig. 4. Schemes of OTR optics removing the small-angle photons for improving the spatial resolution: (a) backward OTR with a mask $m$ in the focal plane $f$; (b) forward OTR with a hole in the collecting mirror C. Others symbols are: $\mathrm{R}=$ radiator; $\mathrm{L}=$ focusing lens; $\mathrm{D}=$ photon detector; $\mathrm{I}=\mathrm{OTR}$ profile for one electron. In scheme (a) a mirror can be put in front of the mask to use the small-angle photons for studying the beam divergence (detector $d$ ). Scheme (b) can also be used for backward OTR, just reversing the direction of the electron beam (as in Fig. 1 of Ref. [3]).

$f_{\mathrm{a}}\left(\mathbf{k}_{\mathrm{T}}\right)=\exp \left[-\mathbf{k}_{\mathrm{T}}^{2} /\left(2 q_{1}^{\prime 2}\right)\right]-\exp \left[-\mathbf{k}_{\mathrm{T}}^{2} /\left(2 q_{m}^{\prime 2}\right)\right]$

with cutoffs $q_{m}^{\prime}=1 / b_{m}^{\prime}$ and $q_{1}^{\prime}=1 / b_{1}^{\prime}$. The resulting apodized profile is

$I_{\mathrm{a}}(b)=\frac{\alpha}{\pi^{2} b^{2}}\left|\exp \left[-b^{2} /\left(2 b_{m}^{\prime 2}\right)\right]-\exp \left[-b^{2} /\left(2 b_{1}^{\prime 2}\right)\right]\right|^{2}$.

One may also use Eq. (28) as a practical approximation to the nonapodized formula, Eq. (9) or Eq. (23), for experiments which are not too sensitive to the oscillations and the $b^{-3}$ tail. To keep the photon flux unchanged, the primed cutoffs must be

$b_{1}^{\prime} \simeq 0.75 b_{1}, \quad b_{m}^{\prime} \simeq \begin{cases}0.91 b_{0} & \text { without mask } \\ 1.5 b_{m} & \text { with mask }\end{cases}$
Apodized curves for masked $b^{2} I(\mathbf{b})$ are compared to nonapodized ones in Fig. 3. The corresponding MTF function is also drawn in Fig. 2.

\section{Conclusions}

The above analysis have shown that, in spite of their different geometrical natures, backward and forward OTR not only have the same angular distribution but also give the same impact parameter profile in the image plane of the radiator surface. Backward OTR is best described as reflection of the quasi-real photon cloud, forward OTR is essentially emitted by the electron emerging from the radiator. In both cases the spatial profile ressembles that of the quasi-real photon cloud except for a hole at small $b$ and diffraction 


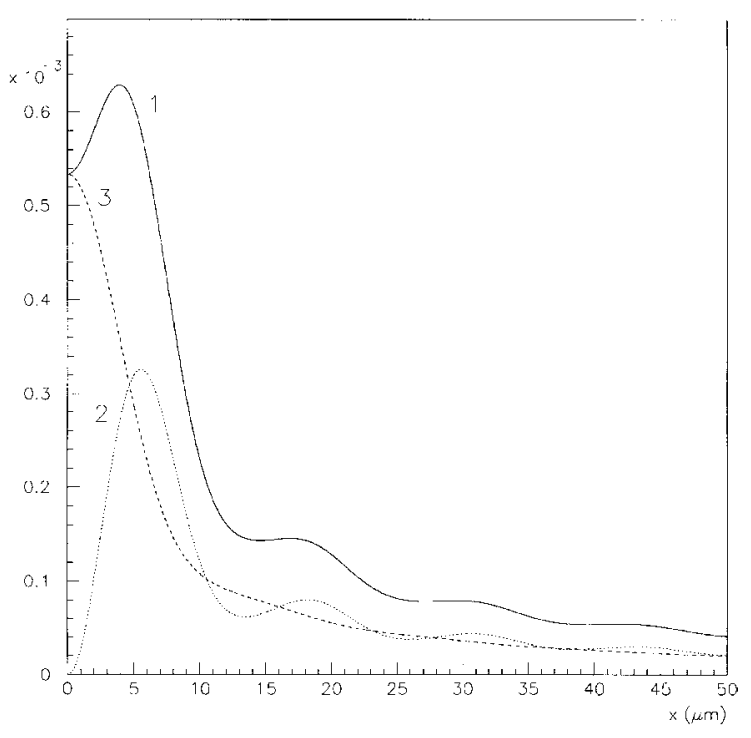

Fig. 5. One-dimensional OTR profile (Eq. (25)) for the natural polarization (curve 1), the $x$-polarization (curve 2) and the $y$ polarization (curve 3). The parameters are the same as in Fig. 1. The vertical scale is in $\mu \mathrm{m}^{-1}$.

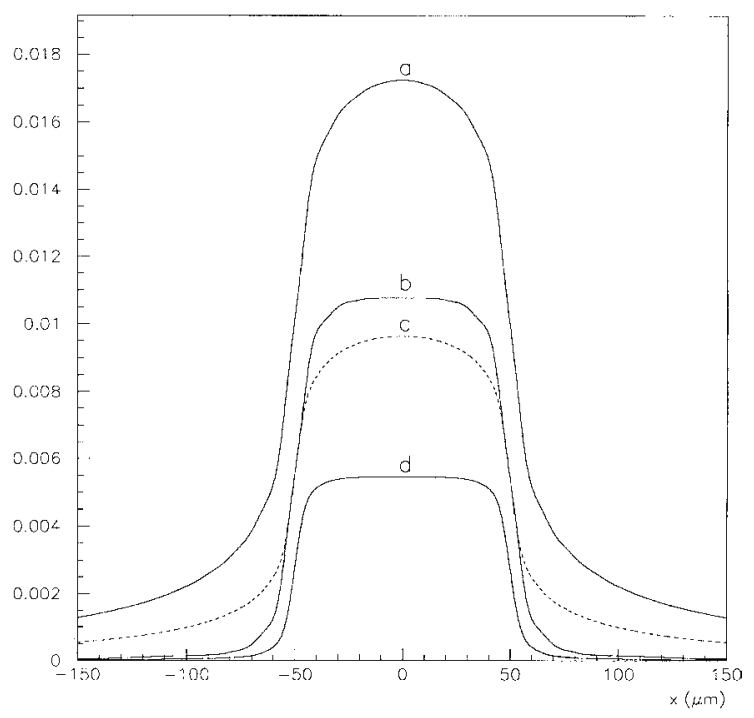

Fig. 6. Convolution of the one-dimensional OTR profile (Eq. (25)) with a window-shaped beam profile $100 \mu \mathrm{m}$ wide: (a) ordinary OTR; (b) OTR with a mask; (c) $y$-polarized OTR; (d) $y$ polarized OTR with a mask. The parameters are the same as in Fig. 3. Edge sizes $\delta r$ (see Section 6) are respectively 19, 11, 14 and $6.5 \mu \mathrm{m}$. fringes at large $b$. Its main features are (i) a minimal distance $b_{1} \sim \chi / \theta_{1}$ imposed by diffraction on the optics, (ii) a maximal distance $b_{0}=\gamma \chi$ given by self-diffraction, (iii) between these two distances, a bulk $b^{-2}$ behavior.

Pure single-surface OTR requires severe conditions at high gamma. As far as the electron trajectory is concerned, it must satisfy a similar formation length condition in the forward and backward cases. If not, the spatial resolution may be improved but one has to consider the interference with one or several other radiators. Concerning the photon collecting system, for forward OTR it must be at distance larger than $l_{\mathrm{f}}(\theta)$ to avoid both formation zone and near-field effects. There is practically no such condition for backward OTR, except if the foil is perpendicular to the beam.

The non-Gaussian shape of the OTR profile makes the resolution power strongly dependent on the type of measurement. For measuring the FWHM width of a beam, self-diffraction caused by the angular peak is not a limitation and ordinary OTR is sufficient. The observation of substructures depends strongly on the contrast sensitivity of the detector and on the beam width itself. For that purpose, and for the detection of a beam halo, great improvements are possible using either a mask removing the small-angle photons, or a polarizer, or both. Apodization of the mask and diaphragm may bring some additional improvement.

\section{Note added in proof}

After completion of this work, we have received a paper by Castellano and Verzilov [15], treating the same questions, with somewhat different formalism, and reaching basically the same conclusions.

\section{References}

[1] K.T. Mc Donald, D.P. Russel, Proc. of Joint US-CERN School in Observation, Diagnosis and Correction on Particle Beams, Capri, Italy, 20-26 October 1988. 
[2] D.W. Rule, R.B. Fiorito, AIP Conference Proceedings 229 (1991) 315; Proc. of 1993 Particle Accelerator Conference, Washington, DC, May 1993, p. 2453.

[3] E.W. Jenkins, Single Pass Collider Memo, 260, (1983).

[4] V.A. Lebedev, Nucl. Instr. and Meth. A 372 (1996) 344.

[5] X. Artru et al., Proc. Fifth European Particle Accelerator Conf., Sitges, June 1996, p. 1686; X. Artru et al., Nucl. Instr. and Meth. A 410 (1998) 148.

[6] D. Giove et al., Proc. of DIPAC 97, Frascati, Italy, 12-14 october 1997.

[7] J.-C. Denard et al., Proc. of 1997 Particle Accelerator Conference, Vancouver, Canada, May 1997.

[8] L. Wartski, Ph.D. Thesis, Université Paris-Sud (1976).

[9] V.E. Pafomov, Proc. Lebedev Inst. of Physics 44 (1971) 25.
[10] P. Rullhusen, X. Artru, P. Dhez, Novel Radiation Sources Using Relativistic Electrons, World Scientific, Singapore, 1998.

[11] S.D. Borovkov et al., Nucl. Instr. and Meth. A 294 (1990) 101.

[12] V. Berestetskii, E. Lifshitz, L. Pitayevskii, Quantum Electrodynamics, Pergamon Press, New York, 1982.

[13] J.D. Jackson, Classical Electrodynamics, Wiley, New York, 1975.

[14] W. Panofsky, M. Philipps, Classical Electricity and Magnetism, Addison-Wesley, Reading, MA, 1962.

[15] M. Castellano, V.A. Verzilov, preprint LNF-98/017 P, July 1, 1998. 